\title{
Perfil epidemiológico do escorpionismo em crianças no estado de Pernambuco, 2015-2019
}

RESUMO | Objetivo: Analisar o perfil epidemiológico do escorpionismo em crianças, no Estado de Pernambuco, nos anos de 2015 a 2019. Métodos: Trata-se de um estudo ecológico, de caráter exploratório, cujo dados foram extraídos do Sistema de Informação de Agravos de Notificação (fichas de notificação preenchidas no período de 2015 a 2019). A análise dos dados foi realizada por meio do Statistical Package for the Social Sciences versão 20.0. Resultados: Entre 2015 e 2019 foram registrados 17.825 acidentes com escorpiões em crianças de até 14 anos no Estado de Pernambuco. A faixa etária mais acometida foi a de 5 a 9 anos (32,70\%). Crianças de 0 a 4 anos de idade apresentaram duas vezes mais chances de apresentar quadro clínico grave (p: <0,001; OR: 2,353; IC95\%:1,650 - 4,782). Conclusão: Houve uma elevada taxa de agravos no período analisado, especialmente em crianças em início de vida escolar, suscitando medidas de prevenção mais eficazes.

Palavras-chaves: Criança; Epidemiologia; Picadas de escorpião; Sistemas de informação em Saúde.

ABSTRACT | Objective: To analyze the epidemiological profile of scorpionism in children, in the State of Pernambuco, in the years 2015 to 2019. Methods: This is an ecological, exploratory study, whose data were extracted from the Notifiable Diseases Information System (data sheets). notification completed from 2015 to 2019). Data analysis was performed using the Statistical Package for the Social Sciences version 20.0. Results: Between 2015 and 2019 there were 17,825 accidents involving scorpions in children up to 14 years old in the State of Pernambuco. The most affected age group was 5 to 9 years old (32.70\%). Children from 0 to 4 years of age were twice as likely to have a severe clinical condition (p: <0.001; OR: 2.353; 95\% Cl: 1.650 - 4.782). Conclusion: There was a high rate of injuries in the analyzed period, especially in children at the beginning of school life, giving rise to more effective prevention measures. Keywords: Child; Epidemiology; Scorpion stings; Health information systems.

RESUMEN | Objetivo: Analizar el perfil epidemiológico del escorpionismo en niños, en el estado de Pernambuco, en los años 2015 a 2019. Métodos: Este es un estudio exploratorio ecológico, cuyos datos fueron extraídos del Sistema de Información de Enfermedades Notificables (fichas técnicas). notificación completada de 2015 a 2019). El análisis de los datos se realizó utilizando el Statistical Package for the Social Sciences versión 20.0. Resultados: Entre 2015 y 2019 se produjeron 17,825 accidentes con escorpiones en niños de hasta 14 años en el estado de Pernambuco. El grupo de edad más afectado fue el de 5 a 9 años (32,70\%). Los niños de 0 a 4 años tenían el doble de probabilidades de tener una enfermedad clínica grave (p: <0,001; OR: 2,353; IC del 95\%: 1,650 - 4,782). Conclusión: Hubo una alta tasa de lesiones en el período analizado, especialmente en niños al inicio de la vida escolar, dando lugar a medidas de prevención más efectivas.

Palabras claves: Niño; Epidemiología; Picaduras de Escorpión; Sistemas de Información en Salud.

\section{Natália Borba Cavalcanti}

Enfermeira graduada pela Universidade Católica de Pernambuco.

ORCID: 0000-0001-7052-8490

\section{Ana Carla Macedo da Silva}

Enfermeira graduada pela Universidade Católica de Pernambuco.

ORCID: 0000-0003-0521-8909

\section{José William Araújo do Nascimento}

Enfermeiro (Universidade Católica de Pernambuco), Mestrando em Ciências da Computação (Informática Médica) pela Universidade Federal de Pernambuco e Sócio da Sociedade Brasileira de Anatomia. ORCID: 0000-0002-1844-1117

\section{Fernando Ramos Gonçalves}

Enfermeiro (Universidade Federal de Pernambuco), Residência em Medicina Preventiva e Social (FIOCRUZ), Mestre em Saúde Pública (CENTRO DE PESQUISA AGGEU MAGALHÃES / FIOCRUZ), Doutorando em Ciên- cias das Linguagens (Universidade Católica de Pernambuco), Docente da FENSG-UPE e Universidade Católica de Pernambuco (Enfermagem e Medicina), Enfermeiro intensivista da Unidade de Suporte Avançado em Neurocirurgia-USAN/Hospital da Restauração e Preceptor da Residência de Enfermagem em Terapia Intensiva e Residência de Enfermagem em Neurologia/Neurocirurgia. ORCID: 0000-0003-2692-9769

\section{Steffany de Almeida Ferreira}

Biomédica (Universidade Federal de Pernambuco), Enfermeira (Universidade Federal de Pernambuco), Especialista em Enfermagem em Saúde da Mulher com ênfase em Ginecologia e Obstetrícia, Mestre e Doutora em Inovação Terapêutica (Universidade Federal de Pernambuco), Enfermeira do Hospital da Restauração de Pernambuco (HR), Enfermeira do PROCAPE e Professora Assistente I do curso de Bacharelado em Enfermagem da Universidade Católica de Pernambuco (UNICAP); ORCID: 0000-0003-2357-3146
Recebido em: 26/01/2021

Aprovado em: 09/02/2021

INTRODUÇÃO

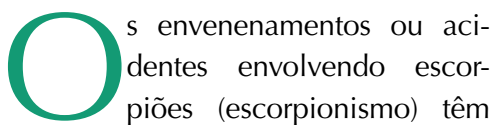
sido considerado um problema de saúde pública em países tropicais e subtropicais, especialmente no Brasil, devido à elevada incidência e a potencialidade do veneno de algumas espécies em provocar situações clínicas graves, muitas vezes fatais ${ }^{(1-4)}$.

Estes acidentes vêm crescendo a cada ano devido principalmente à modificação do ambiente natural das espécies, aos locais propícios a sua adaptação como as redes hidrográficas, ocupações irregulares, áreas verdes e presença ou ausência de saneamento básico além das espécies de escorpião ter sua reprodução 
por partenogênese, o que colabora na proliferação destes seres ${ }^{(4)}$.

Estima-se que, anualmente, aproximadamente 1,5 milhão de acidentes por escorpião ocorram no mundo, provocando cerca de 2.600 óbitos, especialmente nos países tropicais ${ }^{(5)}$. A nível brasileiro, dados oficiais do Ministério da Saúde apontam que cerca de 50 mil casos de acidentes desta natureza ocorra anualmente, principalmente nos períodos mais quentes, fazendo com que os casos de escorpionismo já superem os de ofidismo ${ }^{(6,7)}$.

Muitos destes acidentes envolvem crianças e de acordo com dados do Sistema de Informação de Agravos de Notificação (SINAN), entre os anos de 2010 e 2014, foram registrados 16.183 e 24.521 casos de intoxicação entre crianças e adolescentes no Brasil. Referindo-se especificamente aos casos de escorpionismo, este foi responsável por $46,6 \%$ dos referidos casos. No Estado de Pernambuco, neste mesmo período de 2010 a 2014, o Centro de Assistência Toxicológica (CEATOX) registrou números que passaram de 2.316 para 4.585 notificações ${ }^{(8)}$.

Diante da crescente quantidade destes acidentes notificados e subnotificados entre crianças, torna-se oportuna a análise retrospectiva de dados envolvendo escorpionismo, principalmente devido à escassez de estudos relativos a temática e voltados a regiões do Nordeste brasileiro.

A pesquisa conduziu-se sobre a seguinte questão norteadora: Qual o perfil epidemiológico de crianças vítimas do escorpionismo no Estado de Pernambuco entre os anos de 2015 e 2019? A resposta a esta questão trouxe subsídios que podem ser úteis na formulação de estratégias de prevenção e identificação de fatores de risco para este público em questão, uma vez que estudos acerca de acidentes por escorpião nesse âmbito populacional são mais raros.

Desta forma este estudo objetivou traçar o perfil epidemiológico do escorpionismo em crianças do Estado de Pernambuco, referente aos anos de 2015 a 2019.
MÉTODOS

Neste estudo, o perfil epidemiológico foi traçado por meio de um estudo ecológico, de caráter exploratório. A coleta de dados foi realizada entre os meses de janeiro a abril de 2020 por meio do acesso aos dados pertencentes às fichas de notificação depositadas na base de dados do SINAN, referentes ao período de 01 de janeiro de 2015 a 31 de dezembro de 2019, sendo relativas ao Estado de Pernambuco.

O Estado referente a esta pesquisa apresenta uma população estimada de 9.557.071 habitantes, sendo aproximadamente 2.256.769 crianças de 0 a 14 anos, com uma área total de 98.068,021 km², caracterizado por dois tipos de clima: tropical úmido (predominante no litoral); semiárido (predominante no interior) ${ }^{(9)}$.

Para a identificação dos dados encontrados no SINAN foram tomados alguns critérios de elegibilidade. O critério de inclusão proposto foi analisar apenas os dados referentes às crianças de 0 a 14 anos de idade. Por sua vez foram excluídos da análise os casos registrados no Sistema como ignorados para quaisquer uma das variáveis registradas.

As variáveis selecionadas para análise deste estudo foram baseadas em dois eixos: 1) dados sócio demográficos: faixa etária (crianças de 0 a 14 anos de idade), sexo (masculino ou feminino), raça e região de saúde de residência; 2) dados relativos ao acidente: mês de ocorrência, microrregião de notificação (19 microrregiões do Estado de Pernambuco), tempo decorrido entre a picada e o atendimento $(0$ a 1 hora, 1 a 3 horas, 3 a 6 horas, 6 a 12 horas, 12 a 24 horas, mais de 24 horas), a classificação final do acidente (leve, moderado ou grave) e a evolução do caso (cura, óbito por escorpionismo e óbitos por causas diversas).

Para análise estatística dos dados foi utilizado o programa Statistical Package for the Social Sciences (SPSS) versão 20.0. Foram determinados a distribuição de frequências absolutas e relativas, sendo realizada uma análise univariada 
dos dados coletados por meio do teste qui-quadrado (X2), para comparação de proporções, adotando-se o nível de significância de $p<0,05$. As estimativas de risco foram calculadas pelo Odds Ratios

(OR) e o intervalo de confiança utilizado foi de 95\% (IC 95\%). A análise estatística foi realizada excluindo-se o valor absoluto das variáveis ignoradas, de forma a permitir uma maior precisão dos dados.
Figura 1: Distribuição percentual dos casos de escorpionismo em crianças no Estado de Pernambuco (Brasil), no período 2015-2019

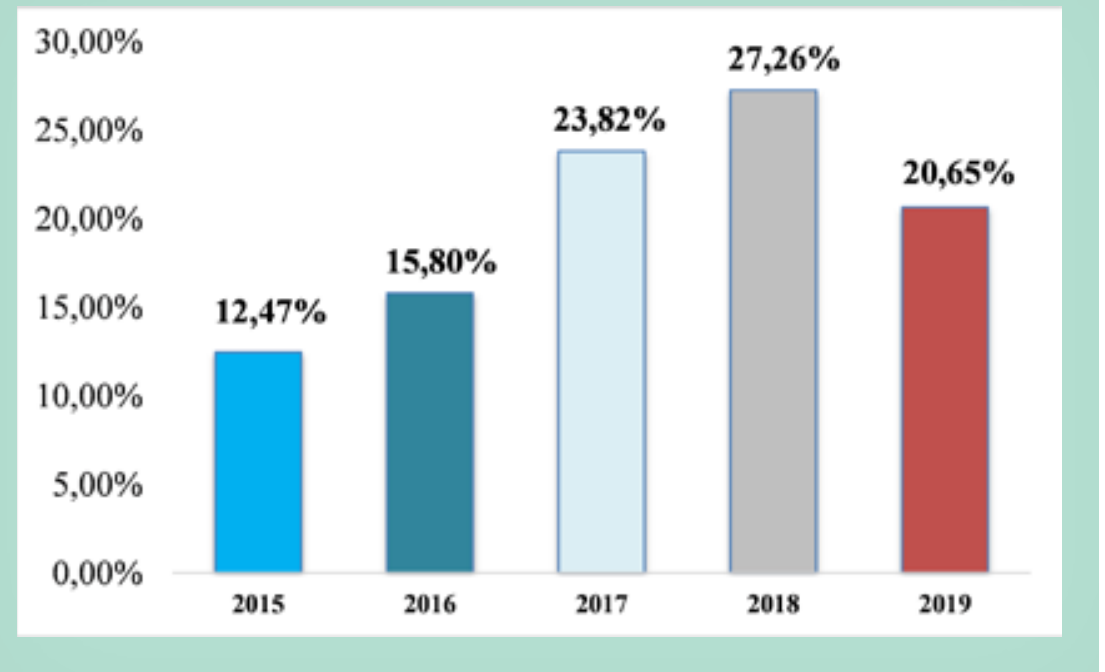

Fonte: Dados extraídos do SINAN, 2020.

Tabela 1: Medidas de frequência das características sociodemográficas das vítimas infantis do escorpionismo, referentes ao estado de Pernambuco (Brasil), no período 2015-2019.

\begin{tabular}{lcc}
\multicolumn{1}{l}{ Variável* } & Frequência absoluta & $\%$ \\
Faixa etária & & \\
\hline$<1$ Ano & 659 & 6,76 \\
\hline $1-4$ & 2.815 & 28,86 \\
\hline $5-9$ & 3.188 & 32,70 \\
$10-14$ & 3.089 & 31,68
\end{tabular}

Gênero

\begin{tabular}{lcc} 
Masculino & 8.949 & 50,20 \\
\hline Feminino & 8.876 & 49,80 \\
\hline Raça & & \\
\hline Amarela & 56 & 0,32 \\
\hline Indígena & 148 & 0,83 \\
\hline Preta & 281 & 1,57 \\
\hline Branca & 1.364 & 7,65 \\
\hline Parda & 7.636 & 42,83
\end{tabular}

Fonte: Dados extraídos do SINAN, 2020.

Nota: * Não foram computadas as variáveis ignoradas ou em branco.
Esta pesquisa seguiu as diretrizes dispostas na Resolução 196/96 do Conselho Nacional de Ética em Pesquisa e por se tratar de um estudo que utiliza dados secundários, não existiu implicações éticas envolvidas, não necessitando de análise pelo comitê de ética e pesquisa.

\section{RESULTADOS}

No período analisado (2015-2019), de acordo com os dados disponibilizados pelo SINAN, foram registrados 17.825 acidentes com escorpiões em crianças de até 14 anos no Estado de Pernambuco, de modo que o ano de 2018 apresentou a maior frequência de casos, representando $26 \%$ do total. A distribuição percentual de escorpionismo neste período está representado na Figura 1.

A faixa etária de maior incidência foi a de 5 a 9 anos ( $n$ : 6.036 - 32,70\%) enquanto a maior parte dessas crianças eram do gênero masculino ( $\mathrm{n}$ : 8.949 - 50,20\%) e de raça parda (n: $7.636-42,83 \%$ ).

Referente ao mês de ocorrência do escorpionismo em Pernambuco, aqueles que registraram maior frequência do acidente foi agosto (n: 1.815) e setembro (n: 1.787). O mês de menor ocorrência no período analisado foi março (n: 1.242). Das 19 microrregiões de notificação do Estado, a que teve uma maior incidência de casos foi Recife (n: 8.773) seguido do Vale do Ipojuca (n: 1.412). O menor índice foi registrado em Fernando de Noronha (n: 01).

Consoante a Tabela 2, maior parte das crianças vítimas do escorpionismo foram atendidas em até 06 horas (79,50\%), principalmente até 01 horas $(45,78 \%)$. Do total de casos, 15.445 (86,64\%) foram registrados como leve, porém, mesmo assim não é desprezível o número de casos classificados como moderado e grave.

Cerca de 16.102 casos (90,33\%) evoluíram para cura. Nos 5 anos avaliados neste estudo, 07 mortes foram registradas em crianças pernambucanas, sendo cinco resultados do efeito direto da picada do escorpião. 
A faixa etária foi considerada como possível variável de exposição para o evento "caso grave". Crianças de 0 a 4 anos de idade apresentaram duas vezes mais chances de terem quadro clínico grave quando comparadas com às de 5 a 14 anos (Teste do qui-quadrado - comparando duas variáveis dicotômicas) (Tabela 3).

\section{DISCUSSÃO}

Com base nos dados extraídos do SINAN, uma elevada prevalência de acidentes envolvendo escorpiões em crianças foi registrada no período de 2015 a 2019, com pico no ano de 2018. Neste ano houve índices pluviométricos acima da média em Pernambuco, em comparação com os demais anos da amostra, especialmente no primeiro trimestre(10). O pico pode ser justificado pelo fato das fortes precipitações serem conjuntas com uma baixa umidade do ar, o que provoca abrigo de escorpiões em locais aquecidos das residências, asso-

ciado ao fato das crianças objetivarem estes lugares em busca de diversão(11).

Maior parte dos acidentes foram registrados nos meses de agosto a setembro, com exceção do pico em julho, no ano de 2018. Uma forte correlação entre picadas de escorpião e a sazonalidade tem sido descrita em estudos de diferentes regiões do mundo ${ }^{(12)}$, incluindo o Sudeste do Brasil ${ }^{(13)}$ e especialmente o Nordeste brasileiro ${ }^{(11,14)}$.

Com relação à região de ocorrência, maior parte dos acidentes ocorreram na capital do Estado (Recife), incluindo toda a região metropolitana. A maior incidência de escorpionismo infantil nessa região justifica-se, pois, áreas urbanas de alta densidade são locais onde existe um alto risco de picadas de escorpião(15). Dados semelhantes foram encontrados em estudos realizados na região metropolitana do Ceará entre 2003 a 2004 ${ }^{(15)}$, bem como em Pernambuco (2007-2010), onde 53,3\% das vítimas infantis eram de Recife $^{(11)}$.

Tabela 2: Medidas de frequência relativas ao tempo decorrido entre a picada e o atendimento e classificação final das vítimas, referentes ao Estado de Pernambuco (Brasil), no periodo 2015-2019.

Variável* Frequência absoluta $\%$

Tempo entre picada e atendimento

\begin{tabular}{|ccc|}
\hline $0-6 \mathrm{~h}$ & 14.172 & 79,50 \\
\hline $6-12 \mathrm{~h}$ & 350 & 1,96 \\
\hline Mais de $12 \mathrm{~h}$ & 408 & 2,28 \\
\hline Classificação final & & \\
\hline Leve & 15.445 & 86,64 \\
\hline Moderado & 538 & 3,01 \\
\hline Grave & 160 & 0,89 \\
\hline
\end{tabular}

Fonte: Dados extraídos do SINAN, 2020.

Nota: * Não foram computadas as variáveis ignoradas ou em branco.

Tabela 3: Casos graves de acordo com a faixa etária, referentes ao Estado de Pernambuco (Brasil), no período 2015-2019.

\begin{tabular}{ccccccc} 
I & \multicolumn{2}{c}{ Casos grave } & Total & $\mathbf{p}^{*}$ & OR ** & III \\
Idade & Não & $\operatorname{Sim}(\%)$ & & & & \\
\hline 0-4 anos & 5.910 & $100(1,66)$ & 6.010 & $<0,001$ & 2,353 & $1,650-4,782$ \\
\hline 5-14 anos & 10.802 & $60(0,55)$ & 10.862 & - & - & - \\
\hline
\end{tabular}

Fonte: Dados da pesquisa.

Nota: *p calculado pelo teste do Qui-quadrado; **Odds Ratio; +Intervalo de Confiança de 95\%
À luz dos resultados, a faixa etária mais acometida foi a de 5 a 9 anos, mesmo padrão obtido em uma pesquisa equivalente realizada no Estado da Bahia, relativo a dados do período de 2007 a $2010^{(14)}$. Porém, estes resultados diferem de um estudo referente também ao Estado de Pernambuco, mas relacionado ao período de 2006 a 2010, onde a maior incidência dos casos concentrava-se em crianças menores de 5 anos $(40,9 \%)^{(11)}$.

Destaca-se que a faixa etária está intimamente relacionada com o agravo dos casos. Crianças de 5 a 9 anos geralmente buscam diversão em lugares mais discretos, distante da presença dos pais. Isto pode ter sido um fator contribuinte para a ocorrência dos casos neste estudo. Embora tenha sido encontrada diferenças na frequência de acidentes por faixa etária, quando comparado com outras pesquisas ${ }^{(11,14)}$, ambas se assemelham pela característica climática de seus locais de observância e pelos aspectos sociais de suas populações e ainda assim, as crianças de 0 a 9 anos representaram as maiores vítimas.

Referindo-se ao tempo de atendimento inicial das vítimas, o período de maior incidência foi nas primeiras seis horas, especialmente em até uma hora, o que se justifica pela agilidade no socorro as vítimas bem como pelo acesso rápido a hospitais de referência.

O protocolo de atendimento tanto a nível nacional quanto ao estadual prevê que as vítimas de picadas do escorpião procurem imediatamente uma unidade de saúde como uma Unidade de Pronto Atendimento (UPA), devendo, de acordo com a gravidade do caso, ser encaminhada a um hospital de referência ${ }^{(16)}$.

Resultados apontados por um estudo evidenciaram que crianças atendidas em um tempo superior a três horas apresentaram uma probabilidade de quase duas vezes mais em evoluir a casos graves ${ }^{(17)}$. Outra pesquisa avaliou que para cada hora acrescentada até o atendimento inicial, haveria um acréscimo de $9 \%$ na chance da criança evoluir a óbito por complicações sistêmicas decorrentes ${ }^{(18)}$. 
Em relação à gravidade dos casos conforme faixa etária, a análise estatística evidenciou que crianças de até 4 anos têm duas vezes mais chance de evoluir à forma mais grave em relação às de 5 a 14 anos. Estudos sugerem que crianças de até 4 ou 5 anos têm maior suscetibilidade a forma grave do envenenamento proveniente do escorpião em relação a crianças mais velhas ${ }^{(19,20)}$.

Esta associação deve-se à vulnerabilidade do sistema imunológico e a relação entre a dose do veneno e o peso corporal do paciente, que geralmente são mais baixas nessa faixa etária ${ }^{(21)}$. Estudos demonstram que crianças, sobretudo aquelas de menor idade, por terem menor área corporal, geralmente, apresentam níveis séricos de veneno mais elevados no organismo, assim como, por uma maior absorção do veneno pelo coração e outros órgãos essenciais ${ }^{(22)}$.
Desta forma, para que estes pacientes não evoluam a casos mais graves, programas de prevenção de agravos precisam ser aprimorados e novas estratégias precisam ser traçadas como uma forma de enfrentamento que resulte em taxas menores de acidente bem como controle mais eficaz deste agravo.

\section{CONCLUSÃO}

Verificou-se que no período entre 2015 a 2019, o Estado de Pernambuco registrou elevada prevalência de escorpionismo em crianças, especialmente na faixa etária de 5 a 9 anos. Maior parcela dos casos foram leves e atendidos em até seis horas, evoluindo à cura, sendo registrados apenas sete óbitos no período.

Constatou-se que crianças de até 4 anos de idade têm mais chance de evoluir a complicações mais grave decorren- tes da picada do escorpião, necessitando então que políticas públicas de prevenção voltadas a esta população sejam mais eficazes e constantes.

Com relação às limitações desta pesquisa, por esta ser referente apenas a dados de um Estado da Federação do Brasil, não é possível generalizá-los à todas regiões. Nas fichas de notificação analisadas não existe registros da identificação das espécies de escorpiões causadoras do evento além da falta de informações dos profissionais de saúde relativos a todas as variáveis.

O conteúdo documentado neste estudo pode ser significativo para gestores e assistentes de saúde implementarem programas de prevenção voltados à acidentes envolvendo escorpiões em crianças. Políticas públicas nesta temática precisam ser reforçadas e implementadas de forma a diminuir a incidência desse agravo neste grupo de risco.

\section{Referências}

1. Brasil. Ministério da Saúde. Fundação Nacional de Saúde. Manual de diagnóstico e tratamento de acidentes por animais peçonhentos. 2 ed. Brasília, DF, 2001'.

2. Bochner $R$, Souza CM. Divergences between the Brazilian national information systems for recording deaths from venomous animals. J Venom Anim Toxins Incl Trop Dis. [Internet]. 2019; 25: e143018.

3. Dourado FS, Bertani R, Cupo P, França FOS. Scorpionism in Brazil: exponential growth of accidents and deaths from scorpion stings. Rev. Soc. Bras. Med. Trop. [Internet]. 2019; 52: e20180350.

4. Pinto GFSG, Pessoa AM, Silva-Jr NJ. Acidentes com escorpiões nas capitais brasileiras entre 2007 e 2014. Estudos. [Internet]. 2015; 42 (4): 539-46.

5. Chippaux JP. Emerging options for the management of scorpion stings. Drug Des Devel Ther. [Internet]. 2012; 6:165-73.

6. Brasil. Ministério da Saúde. Secretaria de Vigilância em Saúde. Manual de Controle de Escorpiões. 1. ed. Brasília, DF, 2009a.

7. Reckziegel GC, Pinto Jr VL. Scorpionism in Brazil in the years 2000 to 2012. J Venom Anim Toxins Incl Trop Dis. [Internet] 2014; 20(46):1-8.

8. Brasil. Ministério da Saúde. Secretaria de Vigilância em Saúde. Sistema de Informação de Agravos de Notificação. Intoxicação exógena e acidente por animais peçonhento, 2014.

9. Instituto Brasileiro de Geografia e Estatística - IBGE، 2019. Resultado dos Dados Preliminares do Censo. 2019.

10. Agência Pernambucana de Águas e Climas - APAC, 2020.

11. Albuquerque CMR, Neto PLS, Amorim MLP, Pires SCV. Pediatric epidemiological aspects of scorpionism and report on fatal cases from Tityus stigmurus stings (Scorpiones: Buthidae) in State of Pernambuco, Brazil Rev Soc Bras Med Trop. [Internet]. 2013; 46(4):484-9.

12. Dabo A, Golou G, Traoré MS, Diarra N, Goyffon M, Doumbo O, et al. Scorpion envenoming in the North of Mali (West Africa): Epidemiological, clinical and therapeutic aspects. Toxicon. [Internet]. 2011; 58:154-58.

13. Barbosa AD, Magalhães DF, Silva JA, Silva MX, Cardoso MFEC, Meneses JNC, et al. Caracterização dos Acidentes Escorpiônicos em Belo Horizon- te, Minas Gerais, Brasil, 2005 a 2009. Cad Saude Publica. [Internet]. 2012; 28:1785-89.

14. Santos JM, Croesy GS, Marinho LFB. Perfil epidemiológico dos acidentes escorpiônicos em crianças, no estado da Bahia, de 2007 a 2010. Rev Enferm Contemp. [Internet]. 2012; 1(1): 118-29.

15. Alves RS, Martins RD, Sousa DF, CD Alves, Barbosa PSF, Queiroz MGR, et al. Aspectos epidemiológicos dos acidentes escorpiônicos no estado do Ceará no período de 2003 a 2004. REPM. 2007; 1: 14-20.

16. Brasil. Ministério da Saúde. Secretaria de Vigilância em Saúde. Departamento de Vigilância Epidemiológica. Manual de controle de escorpiões I Ministério da Saúde, Secretaria de Vigilância em Saúde, Departamento de Vigilância Epidemiológica. - Brasília: Ministério da Saúde, 2009.

17. Horta FMB, Caldeira AP, Seres JAS. Escorpionismo em crianças e adolescentes: aspectos clínicos e epidemiológicos de pacientes hospitalizados. Rev Soc Bras Med Trop. [Internet]. 2007; 40(3):351-53.

18. Bouaziz M, Bahloul M, Kallel H, Samet M, Ksibi H, Dammak H, et al. Epidemiological, clinical characteristics and outcome of severe scorpion envenomation in South Tunisia: Multivariate analysis of 951 cases. Toxicon. [Internet] 2008; 52(8):918-26.

19. Pons AJ, Miranda A, Pedrerol L, Pizzi HL, Páez Rearte MG, Tomas A. Relato de Experiencia: la Xilografía como recurso para aprender sobre escorpionismo en niños de escuelas primarias,en ciudad de Córdoba, Argentina. Rev. salud pública (Córdoba). [Internet]. 2019; 23(2): 78-85.

20. Carmo EA, Nery AA, Pereira R, Rios MA, Casotti CA. Fatores associados à gravidade do envenenamento por escorpiões. Texto Contexto Enferm. [Internet]. 2019; 28:e20170561.

21. Rostagno G, Bonetto G, Saenz S. Escorpionism in pediatric intensive care patients. Case series. Arch Argent Pediatr. [Internet]. 2019; 117(4): E368-E372. 22. Silva-Domínguez $R$, Paredes-Solis $S$, Cortés-Guzmán AJ, Flores-Moreno $M$ Baldazo-Monsivaiz JG, Anderson N, et al. Factors associated with scorpion stings in schoolchildren: cross-sectional study from two rural communities of Guerrero, Mexico. Bol Med Hosp Infant Mex. [Internet]. 2019; 76: 73-80. 